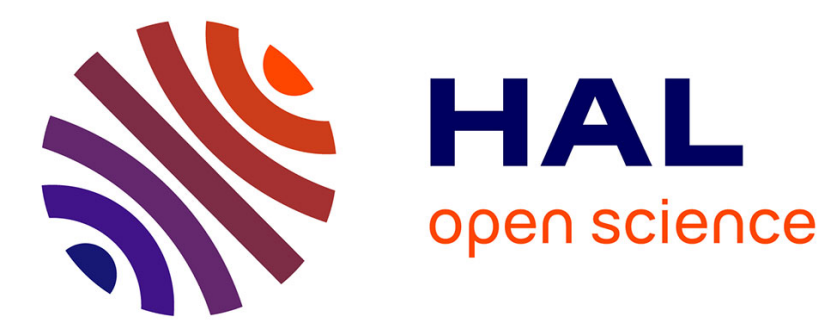

\title{
Sur la décroissance du radium $\mathrm{D}$ \\ Pierre Curie, Irène Curie
}

\section{To cite this version:}

Pierre Curie, Irène Curie. Sur la décroissance du radium D. J. Phys. Radium, 1929, 10 (11), pp.385-

387. 10.1051/jphysrad:019290010011038500 . jpa-00205398

\section{HAL Id: jpa-00205398 https://hal.science/jpa-00205398}

Submitted on 1 Jan 1929

HAL is a multi-disciplinary open access archive for the deposit and dissemination of scientific research documents, whether they are published or not. The documents may come from teaching and research institutions in France or abroad, or from public or private research centers.
L'archive ouverte pluridisciplinaire HAL, est destinée au dépôt et à la diffusion de documents scientifiques de niveau recherche, publiés ou non, émanant des établissements d'enseignement et de recherche français ou étrangers, des laboratoires publics ou privés. 


\title{
LE JOURNAL DE PHYSIQUE
}

\section{LE RADIUM}

\section{SUR LA DÉGROISSANGE DU RADIUM D.}

\author{
Par Mme P. CURIE ET Mme IRÈNE CURIE.
}

\begin{abstract}
Sommaire. - Des observations de la décroissance du RaD poursuivies pendant 16 ans prouvent que la période actuellement admise pour le RaD est trop courte. Le nombre qui résulte de ces expériences est 19.5 ans.
\end{abstract}

Le radium D est une substance dont la vitesse de destruction est faible, et pour mesurer cette vitesse directement il faut, en principe, faire porter les observations sur un grand nombre d'années. La constante radioactive du RaD que l'on admet actuellement, $\lambda=0,0433 \mathrm{ans}^{-1}$, et qui correspond à une période (temps nécessaire pour la diminution de moitié) de 16,5 ans ne résulte pas de l'observation de la décroissance mais de mesures daccroissement de la quantité de polonium qui se forme dans le RaD initialement pur, par suite de sa transformation $\left({ }^{1}\right)$.

L'une de nous a signalé dans une publication antérieure les résultats d'une série de mesures portant sur cinq années et concernant l'évolution des rayonnements $\alpha$ et $\beta$ d'un chlorure de plomb radioactif privés de RaE et de polonium par plusieurs cristallisations $\left(^{2}\right.$ ). Depuis cette époque, les mesures ont été poursuivies pendant 15 ans avec le concours du personnel du Laboratoire, alors que le rayonnement $\beta$ et le rayonnement $\alpha$ étaient en voie de décroissance régulière. On sait que le rayonnement $\beta$ et le rayonnement $\alpha$ sont émis respectivement par le $\mathrm{RaE}$ et par le polonium qui dérivent du RaD et dont la proportion à celui-ci devient constante quand l'équilibre de régime a été établi.

Le chlorure de plomb radioactif était étalé en couche régulière dans une cuvette très peu profonde de $10 \mathrm{~cm}$ de diamètre. Cette cuvette était placée dans une chambre d'ionisation utilisant complètement le parcours des rayons $\alpha$ issus de la surface. On mesurait le rayonnement $\beta$ en recouvrant la cuvette d'un écran d'aluminium de 3/100 de mm d'épaisseur, juste suffisant pour arrêter les rayons $x$ du polonium. Soit $J$ le courant de saturation que l'on mesure sans écran, $J_{3}$ le courant que l'on mesure avec l'écran. La différence $J_{-} J_{3}$ représente sensiblement le courant $\mathcal{J}_{\alpha}$ attribuable aux rayons $\alpha$ du polonium (en négligeant la correction de 1 pour cent environ sur $\mathscr{J}_{\alpha}$, relative à l'absorption des rayons $\beta$ par l'écran).

Les courants ${ }^{3}-\mathcal{J}_{3}$ étaient mesurés en valeur absolue par la méthode du quartz piézoélectrique et on procédait de temps en temps à la vérification de l'étalonnage des lames employées; les courants mesurés étaient au début de l'ordre de $1 \mathrm{ES}$ pour les rayons $\approx$. et de 0,1 ES pour les rayons $\beta$.

D’après les mesures, on a construit une courbe représentant la variation de $\log \mathscr{J}_{\alpha}$ en fonction du temps $t$. Pour $\mathfrak{J}_{9}$ il a été nécesssaire de tenir compte de ce que le courant d'ionisation augmente avec la densité de l'air dans la chambre, densité variable, selon les jours avec la température et la pression; on a admis que le courant est proportionnel à cette densité et l'on a calculé pour chaque mesure une correction $\varepsilon$ telle que $\mathscr{J}_{\beta}^{\prime}=(1+\varepsilon) J_{\xi}$ repré-

(') Avronof. Phil. Mag., t. 19 (1910), p. 852.

(2) $\mathrm{M}^{-}$P. Curik. Le Radium, t. 8 (1911), p. 3 3 3 .

le journal de Physique et le radius. - Série vi. - T. X. - No 11. - Novembre 1929. 
sente le courant qu'on aurait mesuré si la chambre était remplie d'air à la pression normale et à la température de $15^{\circ} \mathrm{C}$; on a construit ensuite la courbe qui représente $\log _{\mathcal{J}^{\prime}}$ en fonction du lemps.

D'avril 1910 à janvier 1926 . les courants $\mathscr{y}_{\%}$ et $\mathfrak{J}^{\prime}$, ont subi une diminution d'environ 40 pour 100. Cependant, on a reonnu au cours des années, que les courbes représentatives, ne sont pas exactement des droites, mais présentent une courbure à peine visible indiquant la présence probable d'une petite quantité d'un radioélément à vie longue produisant des rayons $\alpha$ et des rayons $\beta$. Nous avons pensé que cette impureté pouvait être du radium qui serait resté avec le plomb radioactif malgré le traitement de purification effectué en 1906. Nous avons donc décidé en 1926 d'arrêter l'expérience et d'éclaircir le point douteux en vue d'une correction à déterminer; cette solution paraissait d'autant plus nécessaire qu'il y avait une différence certaine entre les périodes apparentes déduites de $\mathcal{J}_{0}$ et $\mathcal{J}_{3}^{\prime}(22$ ans et 24 ans respectivement).

Pour rechercher si le chlorure de plomb radioactif contenail du radium, on en a prélevé $0, \leftrightarrows$ gr que l'on a transformé en carbonate et dissous dans l'acide nitrique dilué ; la solution a été introduite dans un barboteur et on a şvérilié la présence du radium par la mesure du radon dégagé. La teneur en radium était $4,43 \times 10^{-8}$ gr $\mathrm{Ra}$ par gramme de chlorure de plomb.

Pour évaluer l'influence de cette impureté, on a incorporé du radium à un chlorure de plomb pur et inactif ; la teneur en radium pour cet échantillon a été déterminée comme précédemment, puis le rayonnement $\alpha$ et le rayonnement $\beta$ ont été mesurés exactement dans les mêmes conditions que dans l'expérience principale. On a ainsi trouvé que le courant dû au radium accompagné du radon et des corps $\mathrm{Ra}(\mathrm{A}+\mathrm{B}+\mathrm{C})$, représentait en 1910, $7, \mathfrak{b}$ pour 100 de $J_{\%}$ et 4 à $\breve{5}$ pour 100 de $J_{3}$. Il faut cependant remarquer que la correction qui résulte de la présence de radium n'est pas constante au cours du temps mais augmente

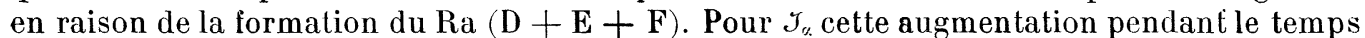
de l'expérience reste inférieure à un dixième du terme correctif initial ; pour $J_{3}$ l'augmentation est difficile à apprécier mais pourrait être de l'ordre de un quart.

Toute discussion faite et compte tenu de la correction, la mesure de $J_{\alpha}$ conduit à une période comprise entre 19 et 20 ans. La mesure de $\mathscr{J}_{3}$ donnerait plutòt une période de 20 à $\mathbf{1}$ ans, mais la courbe est moins bonne et la correction très incertaine.

Les expériences décrites ci-dessus conduiraient à admettre pour la période du RaD

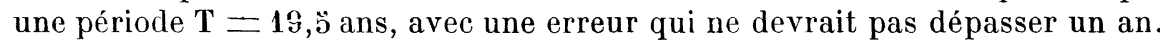

Nous avons recueilli par ailleurs un renseignement complémentaire sur la période du RaD d'après l'étude de l'évolution du rayonnement $\alpha$ de préparations de $\mathrm{Ra}(\mathrm{D}+\mathrm{E}+\mathrm{F})$ provenant d'anciennes ampoules de radon et par conséquent exemptes de radium. La durée totale de ces observations n'étant encore que de כ̆ ans 1/2, on ne peut en tirer de conclusions précises. Les courbes qui représentent l'intensité du rayonnement $\alpha$ en fonction du temps comprennent une partie ascendante, un maximum et la décroissance ultérieure. Pour utiliser au mieux la totalité de la courbe on a employé la forme de calcul suivante :

Désignons par $\lambda_{1}$ et $\lambda_{2}$ respectivement, les constantes radioactives du $\mathrm{RaD}$ et du Po et par $\tau$ l'intervalle de temps qui sépare des valeurs égales de l'ordonnée, de part et d'autre du maximum; soit $k$ le rapport de cette ordonnée à l'ordonnée maximum. On peut établir Ia relation

$$
k=\frac{1}{1-\frac{\lambda_{1}}{\lambda_{2}}}\left[\frac{\lambda_{2}}{\lambda_{1}}\left(\mathbf{e}_{1 \tau}-1\right)\right]^{\frac{1}{\lambda_{1}}-1} \mathbf{e}^{-\frac{\lambda}{1-\frac{\lambda}{\lambda_{2}}}}
$$

La constante radioactive du RaE ne se trouve pas dans cette formule car des approximations légitimes la font disparaître.

Ayant déterminé avec soin un point sur la partie lentement descendante de la courbe, on s'en sert pour déterminer l'intervalle ₹ en cherchant le point de même ordonnée sur la 
partie ascendante à montée rapide; $:$ peut ainsi être déterminé avec précision. 1 cette valeur de $\tau$ correspondra, selon la formule, une valeur de $k$ qui devra concorder avec la valeur expérimentale si la valeur adoptée pour $\lambda_{1}$ est conforme à la valeur réelle. On recherche

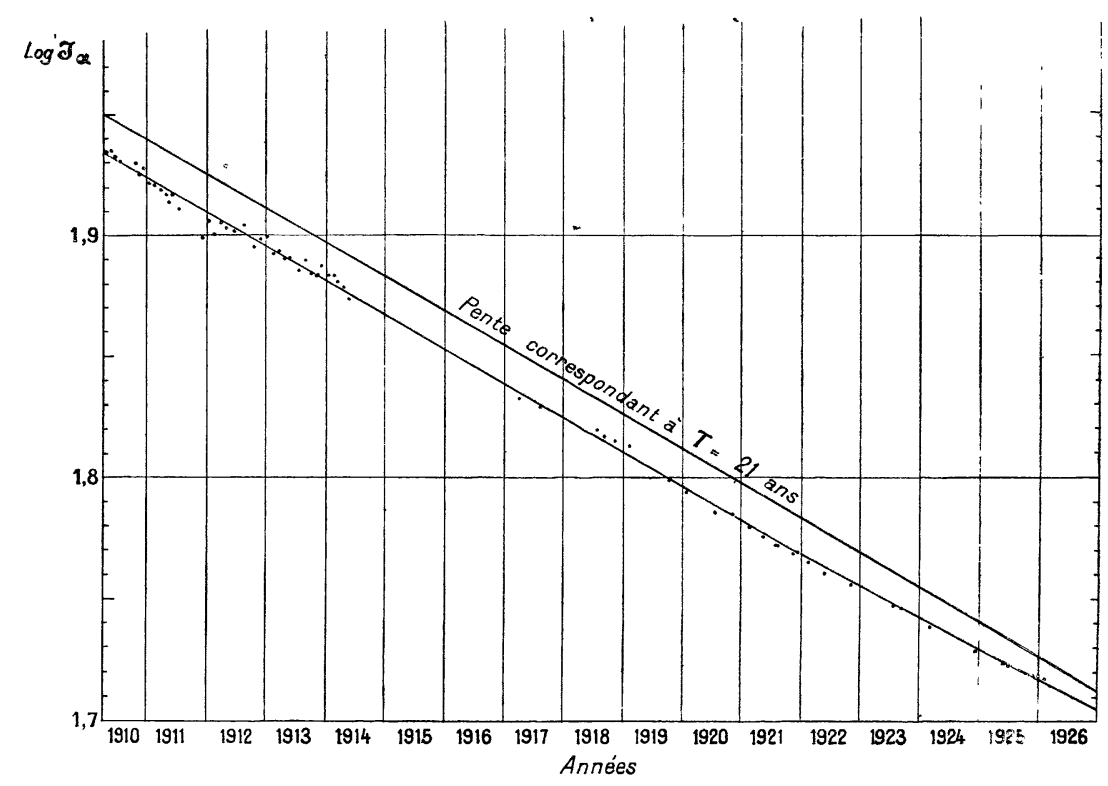

La courbe ci-dessus représente la décroissance de $\log \mathscr{J}_{\sigma}$ en fonction du temps ;

la droite tracée au-dessus de la courbe correspond à la pente initiale de celle-ci et à une période dc 21 ans.

par tàtonnements la valeur de $\lambda_{1}$ qui donne la meilleure concordance. Dans le cas considéré,

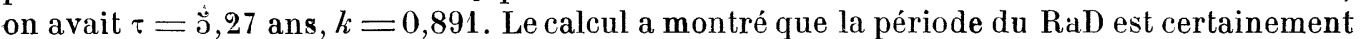
supérieure à la valeur actuellement admise et qu'elle est comprise entre 19 et 24 ans.

On peut conclure que la valeur actuellement adoptée pour la période du RaD est trop faible et que la valeur réelle est au moins égale à 19 ans. Cette conclusion n'est pas en désaccord avec la mesure d'accroissement du débit calorifique d'ampoules de radium anciennes parformation de polonium $\left(^{1}\right)$.

(') Mme P. Curie et D.-K. Yovanovitch. J. de Phys. et Rad., t. 6 (192乡), p. 33. 